\title{
Plastic collapse mechanisms in thin disks subject to thermo-mechanical loading
}

\author{
Sergei Alexandrov ${ }^{1}$ and Chinh Pham $^{2^{*}}$
}

\author{
* Correspondence: \\ pdchinh@imech.ac.vn \\ ${ }^{2}$ VAST, Institute of Mechanics, 264 \\ Doi Can, Hanoi, Vietnam \\ Full list of author information is \\ available at the end of the article
}

\begin{abstract}
Background: A new solution for plastic collapse of a thin annular disk subject to thermo-mechanical loading is presented.

Methods: It is assumed that plastic yielding is controlled by Hill's quadratic orthotropic yield criterion. A distinguished feature of the boundary value problem considered is that there are two loading parameters. One of these parameters is temperature, and the other is pressure over the inner radius of the disk.

Results: The general qualitative structure of the solution at plastic collapse is discussed in detail.

Conclusions: It is shown that two different plastic collapse mechanisms are possible. One of these mechanisms is characterized by strain localization at the inner radius of the disk. The entire disk becomes plastic according to the other collapse mechanism. In addition, two special regimes of plastic collapse are identified. According to one of these regimes, plastic collapse occurs when the entire disk is elastic except its inner radius. According to the other regime, the entire disk becomes plastic at the same values of the loading parameters at which plastic yielding starts to develop.
\end{abstract}

Keywords: Thin disks; Plastic collapse; Plastic anisotropy; Thermo-mechanical loading; Qualitative features of solution

\section{苗


In the present paper, the effect of temperature and pressure over the inner radius of a thin hollow disk on plastic collapse is investigated. The outer radius of the disk is fixed. The state of stress is plane. The classical Duhamel-Neumann law is adopted to connect the thermal and elastic portions of the strain tensor and stress components. Plastic yielding is controlled by Hill's quadratic orthotropic yield criterion [22]. It is assumed that the principal axes of anisotropy coincide with the base vectors of a cylindrical coordinate system $(r, \theta, z)$ whose $z$-axis coincides with the axis of symmetry of the disk. Therefore, the boundary value problem is axisymmetric and its solution is independent of the polar angle. It is shown that the general qualitative structure of the plastic collapse solution is rather complicated. In particular, two different plastic collapse mechanisms have been found. According to one of these mechanisms, the plastic collapse occurs because the entire disk becomes plastic. The other plastic collapse mechanism is characterized by localization of plastic deformation at the inner radius of the disk.

\section{Statement of the problem}

Consider a thin disk of radius $b_{0}$ with a central hole of radius $a_{0}$, which is inserted into a rigid container of radius $b_{0}$ (Figure 1). The disk is subject to thermo-mechanical loading by a uniform pressure $P$ over its inner radius and a uniform increase of temperature $T$ from its initial value. By assumption, both $P$ and $T$ are monotonically increasing functions of a time-like parameter. Also, $P=0$ and $T=0$ at the initial instant. Thus, both $P \geq 0$ and $T \geq 0$. The disk has no stress at the initial instant. It is convenient to introduce a cylindrical coordinate system $(r, \theta, z)$ whose $z$-axis coincides with the axis of symmetry of the disk. Then, the equations for the inner and outer radii of the disk are $r=a_{0}$ and

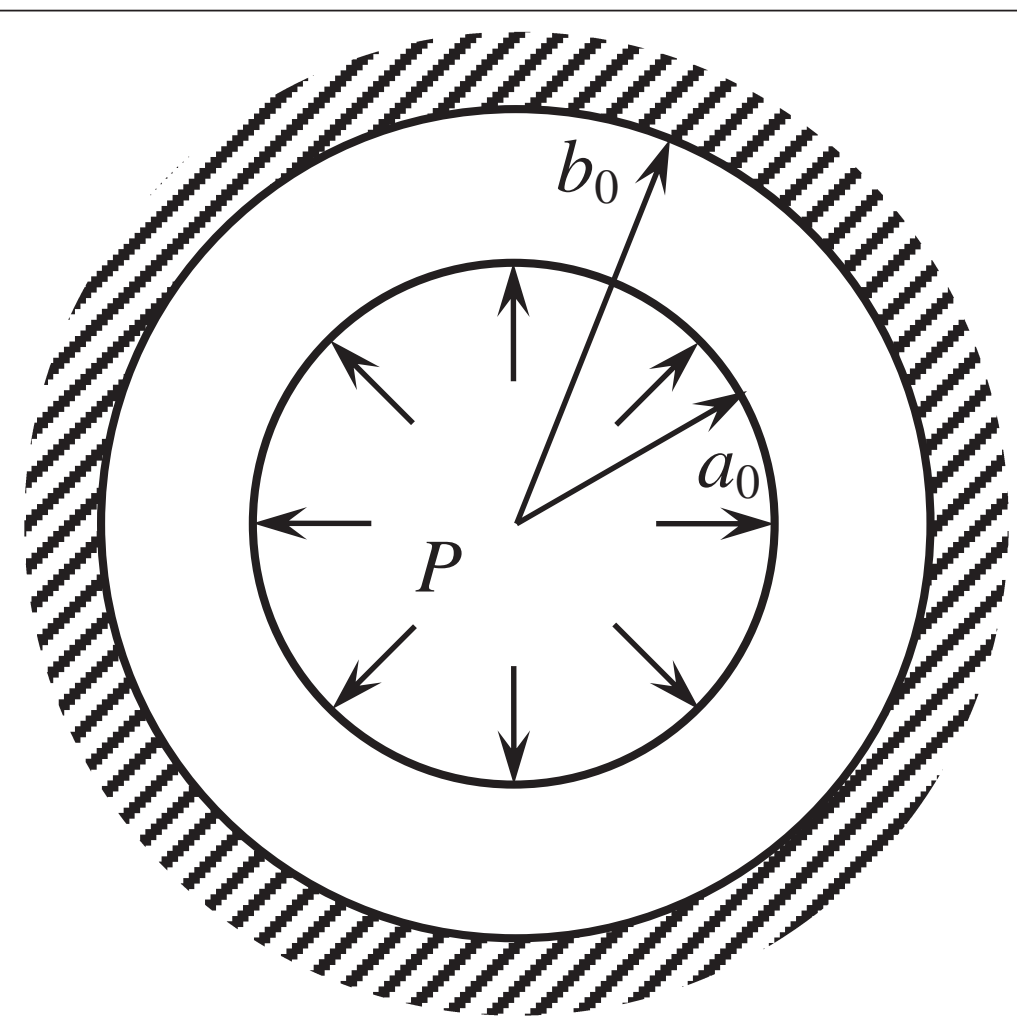

Figure 1 Illustration of the structure. 
$r=b_{0}$, respectively. The strains are supposed to be small. Let $\sigma_{n} \sigma_{\theta}$ and $\sigma_{z}$ be the normal stresses in the cylindrical coordinate system. The state of stress is plane, $\sigma_{z}=0$. It is evident that the problem is axisymmetric. In particular, the solution is independent of $\theta$. Moreover, the normal stresses in the cylindrical coordinates are the principal stresses. The boundary conditions can be written as

$$
\sigma_{r}=-P
$$

for $r=a_{0}$ and

$$
u_{r}=0
$$

for $r=b_{0}$. Here $u_{r}$ is the radial displacement.

The sum of the elastic and thermal portions of the strain tensor follows the classical Duhamel-Neumann law. In the case under consideration, this law reads

$$
\varepsilon_{r}^{e}+\varepsilon_{r}^{T}=\frac{\left(\sigma_{r}-v \sigma_{\theta}\right)}{E}+\alpha T, \quad \varepsilon_{\theta}^{e}+\varepsilon_{\theta}^{T}=\frac{\left(\sigma_{\theta}-v \sigma_{r}\right)}{E}+\alpha T, \quad \varepsilon_{z}^{e}+\varepsilon_{z}^{T}=-\frac{\nu}{E}\left(\sigma_{r}+\sigma_{\theta}\right)+\alpha T
$$

where $\varepsilon_{r}^{e}, \varepsilon_{\theta}^{e}$ and $\varepsilon_{z}^{e}$ are the elastic portions of the strain tensor, $\varepsilon_{r}^{T}, \varepsilon_{\theta}^{T}$ and $\varepsilon_{z}^{T}$ are the thermal portions of the strain tensor, $E$ is Young's modulus, $v$ is Poisson's ratio and $\alpha$ is the thermal coefficient of linear expansion. The plastic portions of the strain tensor will be denoted by $\varepsilon_{r}^{p}, \varepsilon_{\theta}^{p}$ and $\varepsilon_{z}^{p}$. The total strain tensor is the sum of its elastic, thermal and plastic portions. In terms of the radial, circumferential and axial strains, which are also the principal strains, these relations have the form

$$
\varepsilon_{r}=\varepsilon_{r}^{e}+\varepsilon_{r}^{T}+\varepsilon_{r}^{p}, \quad \varepsilon_{\theta}=\varepsilon_{\theta}^{e}+\varepsilon_{\theta}^{T}+\varepsilon_{\theta}^{p}, \quad \varepsilon_{z}=\varepsilon_{z}^{e}+\varepsilon_{z}^{T}+\varepsilon_{z}^{p}
$$

It is assumed that the initiation of plastic yielding is controlled by Hill's quadratic orthotropic yield criterion [22]. By assumption, the principal axes of anisotropy coincide with the base vectors of the cylindrical coordinate system. Therefore, the aforementioned statements that the boundary value problem is axisymmetric and that the principal directions of the stress and strain tensors coincide with the base vectors of the cylindrical coordinate system are justified. Under the assumptions made, the yield criterion becomes

$$
(G+H) \sigma_{r}^{2}-2 H \sigma_{r} \sigma_{\theta}+(H+F) \sigma_{\theta}^{2}=1
$$

where $G, H$ and $F$ are constants which characterize the current state of anisotropy. It is convenient to rewrite (5) as [12]

$$
\sigma_{r}^{2}+p_{\theta}^{2}-\eta \sigma_{r} p_{\theta}=\sigma_{0}^{2}
$$

where

$$
p_{\theta}=\frac{\sigma_{\theta}}{\eta_{1}}, \quad \eta=\frac{2 H}{\sqrt{(G+H)(H+F)}}, \quad \eta_{1}=\frac{\sqrt{G+H}}{\sqrt{H+F}}, \quad \sigma_{0}=\frac{1}{G+H}
$$

No specific relation between stress and strain (or strain rate) in the plastic zone is needed for limit analysis. The only non-trivial equation of equilibrium is

$$
\frac{\partial \sigma_{r}}{\partial r}+\frac{\sigma_{r}-\sigma_{\theta}}{r}=0
$$

Since strains are small,

$$
\varepsilon_{r}=\frac{\partial u_{r}}{\partial r}, \quad \varepsilon_{\theta}=\frac{u_{r}}{r}
$$




\section{Methods}

\section{Elastic solution}

When both $T$ and $P$ are small enough, the entire disk is elastic. For axisymmetric deformation and the plane strain conditions, the equations of linear thermo-elasticity consisting of (3), (8) and (9) have the general solution in the form

$$
\frac{\sigma_{r}}{\sigma_{0}}=\frac{A}{\rho^{2}}+B, \quad \frac{\sigma_{\theta}}{\sigma_{0}}=-\frac{A}{\rho^{2}}+B, \quad k^{-1} u=(1-v) B \rho-(1+v) \frac{A}{\rho}+\tau \rho
$$

where $\rho=r / b_{0}, k=\sigma_{0} / E, \tau=\gamma T E / \sigma_{0}, u=u_{r} / b_{0}$, and $A$ and $B$ are constants of integration. When the entire disk is elastic, $A$ and $B$ are determined from the solution (10) using the boundary conditions (1) and (2) as

$$
A=A_{e}=\frac{a^{2}[\tau-p(1-v)]}{a^{2}(1+v)+1-v}, \quad B=B_{e}=-\left[\frac{(1+v) p a^{2}+\tau}{a^{2}(1+v)+1-v}\right]
$$

where $p=P / \sigma_{0}$ and $a=a_{0} / b_{0}$. As a result of an increase in $\tau$, or $p$ or both, a plastic zone can appear at the inner radius of the disk.

\section{General stress solution in the plastic zone}

In order to find the general stress solution in the plastic zone, it is necessary to combine the yield criterion (6) and the equilibrium equation (8). The yield criterion is satisfied automatically by the following substitution [12]:

$$
\frac{\sigma_{r}}{\sigma_{0}}=\frac{2 \cos \varphi}{\sqrt{4-\eta^{2}}}, \quad \frac{p_{\theta}}{\sigma_{0}}=\frac{\eta \cos \varphi}{\sqrt{4-\eta^{2}}}+\sin \varphi
$$

where $\varphi$ is a function of $\rho$. Substituting (12) into (8) leads to the following ordinary differential equation for $\varphi$ :

$$
2 \sin \varphi \frac{d \varphi}{d \rho}-\left[\frac{2 F \cos \varphi}{(H+F)}-\eta_{1} \sqrt{4-\eta^{2}} \sin \varphi\right] \rho^{-1}=0
$$

The general solution to this equation can be found in elementary functions. However, it is more convenient to write it as

$$
\rho=a \exp \left[\int_{\varphi_{a}}^{\varphi} \frac{d \beta}{\Omega(\beta)}\right], \quad \Omega(\beta)=\frac{F}{(H+F)} \cot \beta-\frac{\eta_{1} \sqrt{4-\eta^{2}}}{2}
$$

where $\beta$ is a dummy variable of integration and $\varphi_{a}$ is the value of $\varphi$ at $\rho=a$.

Consider the mechanism of plastic collapse according to which the plastic zone occupies the entire disk. At the instant of plastic collapse, the elastic zone reduces to the curve $\rho=1$ in the $\rho \theta$-plane. The general solution (10) is valid in this vanishing elastic zone, though $A$ and $B$ are not given by (11). Since the stresses $\sigma_{r}$ and $\sigma_{\theta}$ as well as the displacement $u$ must be continuous across the elastic plastic boundary $\rho=1$, it follows from the boundary condition (2) and Equations (10) and (12) that 


$$
(1-v) B-(1+v) A+\tau=0, \quad A+B=\frac{2 \cos \varphi_{m}}{\sqrt{4-\eta^{2}}}, \quad B-A=\left(\frac{\eta \cos \varphi_{m}}{\sqrt{4-\eta^{2}}}+\sin \varphi_{m}\right) \eta_{1}
$$

where $\varphi_{m}$ is the value of $\varphi$ at $\rho=1$. Using Equation (14) this value is determined in implicit form as

$$
1=a \exp \left[\int_{\varphi_{a}}^{\varphi_{m}} \frac{d \beta}{\Omega(\beta)}\right]
$$

Using (1) and (12) yields

$$
\frac{2 \cos \varphi_{a}}{\sqrt{4-\eta^{2}}}=-p
$$

Taking into account this equation, the solution to the system of Equations (15) and (16) gives a relation between $p$ and $\tau$ when the entire disk becomes plastic. However, a difficulty is that this system may have no solution.

\section{General structure of the solution at plastic collapse}

It will be seen later that the set of parameters at which the plastic zone starts to develop is also of importance for understanding the general structure of the solution at plastic collapse. When plastic yielding begins, the dependence of the radial and circumferential stresses on $\varphi$ is given by Equation (12) at $\rho=a$. Also, the solution (10) with $A$ and $B$ determined from (11) is valid in the range $a \leq \rho \leq 1$. The stresses and the radial displacement must be continuous across the elastic-plastic boundary $\rho=a$. Therefore,

$$
\frac{A_{e}}{a^{2}}+B_{e}=\frac{2 \cos \varphi_{0}}{\sqrt{4-\eta^{2}}}, \quad-\frac{A_{e}}{a^{2}}+B_{e}=\eta_{1}\left(\frac{\eta \cos \varphi_{0}}{\sqrt{4-\eta^{2}}}+\sin \varphi_{0}\right)
$$

where $\varphi_{0}$ is the value of $\varphi_{a}$ at the instant of the initiation of plastic yielding. Since $A_{e}$ and $B_{e}$ are expressed through $p$ and $\tau$, the dependence of $p$ on $\tau$ corresponding to the initiation of plastic yielding is determined from Equation (18). Using the imposed restrictions $p \geq 0$ and $\tau \geq 0$, it is possible to find the range of possible values of $\varphi_{0}$, say $\varphi_{0}^{(1)} \leq \varphi_{0} \leq \varphi_{0}^{(2)}$. A typical dependence of $p$ on $\tau$ corresponding to the initiation of plastic yielding is illustrated in Figure 2. The specific values of parameters used to find this curve are $a=1 / 2, v=0.3$ and $G=H=F$. It is seen from Figure 2 that there is a local maximum of the function $p(\tau)$ at some value of $\tau=\tau_{k}$ (point $k$ in Figure 2). It is evident that $d p(\tau) / d \tau=0$ at $\tau=\tau_{k}$. Replacing $A_{e}$ and $B_{e}$ in Equation (18) by means of Equation (11) and differentiating and excluding $d \varphi_{0}$ lead to

$$
\frac{d p}{d \tau}=\frac{4 \sin \varphi_{0}}{2\left[1-v-a^{2}(1+v)\right] \sin \varphi_{0}+\eta_{1}\left(\eta \sin \varphi_{0}-\sqrt{4-\eta^{2}} \cos \varphi_{0}\right)\left[1-v+a^{2}(1+v)\right]}
$$

It follows from this equation that the condition $d p(\tau) / d \tau=0$ is equivalent to

$$
\varphi_{0}=0
$$




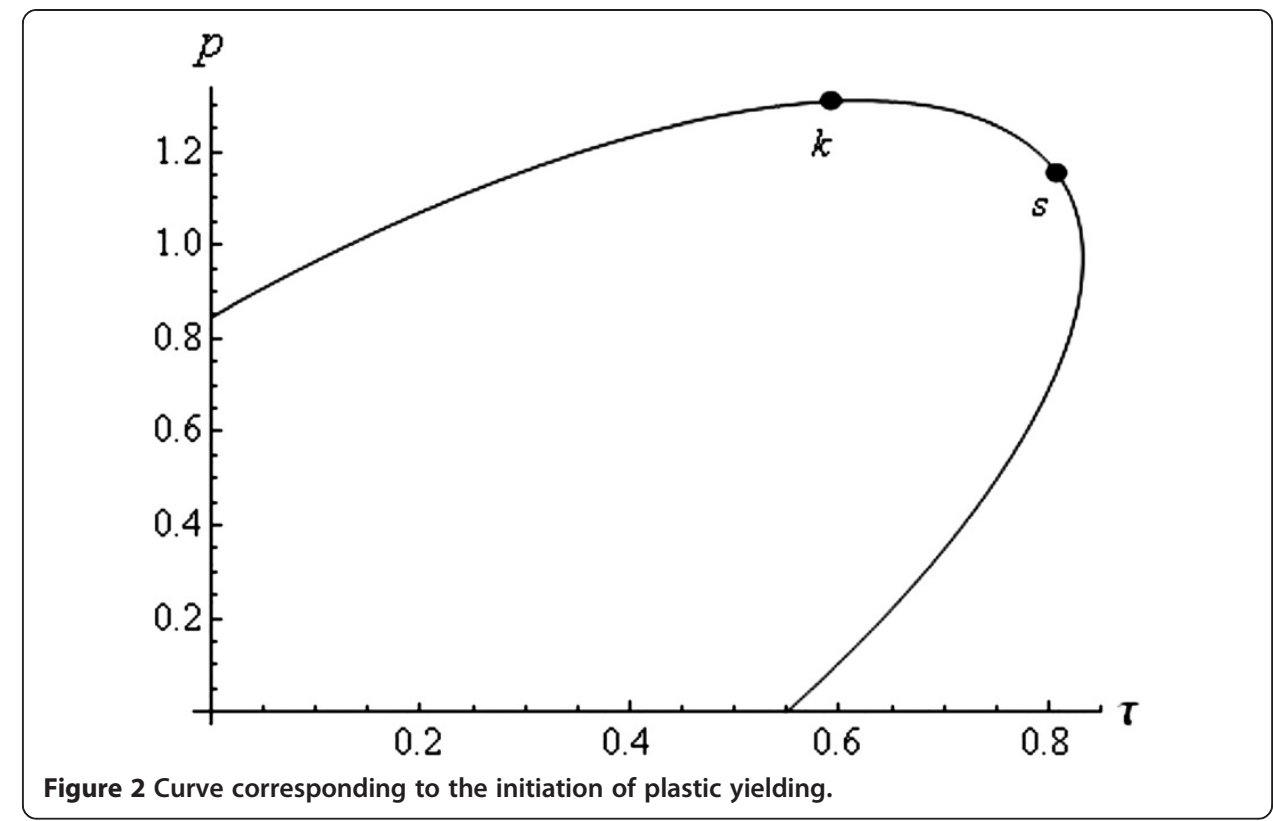

Moreover, it is evident that the coefficient of the derivative in Equation (13) vanishes at $\varphi=\varphi_{0}$ if $\varphi_{0}$ is determined from (20). In the vicinity of the point $\varphi=\varphi_{0}=0$, Equation (13) transforms to

$$
\varphi \frac{d \varphi}{d \rho}-\frac{F}{(H+F) a}=0
$$

Integrating yields

$$
\varphi^{2}=\frac{2 F}{(H+F) a}(\rho-a)
$$

to leading order. It follows from this equation that the plastic zone cannot develop if $\varphi_{0}=0$. For Equation (22) is valid at any elastic/plastic boundary $\rho=\rho_{c}$ at which $\varphi=\varphi_{c}=0$. In this case, the plastic zone occupies the domain $a \leq \rho \leq \rho_{c}$. Therefore, $\rho-\rho_{c}<0$ in the plastic zone, which contradicts the left-hand side of Equation (22). Putting $\rho_{c} \rightarrow a$, it is possible to conclude that this statement is true at the initiation of plastic yielding as well. The physical interpretation of this mathematical feature of the solution is that plastic deformation is localized within a layer of infinitesimal thickness at $\rho=a$. This corresponds to another mechanism of plastic collapse as compared to the state in which the entire disk is plastic. A remarkable property of the set of parameters at point $k$ (Figure 2) is that the disk losses its load-bearing capacity without any plastic deformation in the domain $a<\rho \leq 1$.

Another point of great interest on the curve shown in Figure 2 is that determined by the condition $\varphi=\varphi_{s}$ (point $s$ in Figure 2) where

$$
\tan \varphi_{s}=\frac{2 F}{(H+F) \eta_{1} \sqrt{4-\eta^{2}}}
$$

It is evident that Equation (13) has a special solution $\varphi=\varphi_{s}$ which is not obtainable from (14). Since $\varphi_{s}$ is constant, it follows from (12) that the stresses $\sigma_{r}$ and $\sigma_{\theta}$ are 
independent of $\rho$. The physical meaning of this mathematical feature of Equation (13) is that the plastic zone occupies the entire disk once the plastic zone has initiated at $\rho=a$.

If the initiation of plastic yielding corresponds to any point of the curve shown in Figure 2 other than points $k$ and $s$, then the elastic/plastic boundary propagates from the surface $\rho=a$ until the plastic collapse occurs. As in the special cases considered, the same two plastic collapse mechanisms are possible. In particular, once the value of $\varphi_{a}$ has become zero, the coefficient of the derivative in (13) vanishes. Equations (21) and (22) are valid. Therefore, as before, it is possible to arrive at the conclusion that the solution cannot be extended beyond the value of $\varphi_{a}=0$. The corresponding plastic collapse mechanism is localization of plastic deformation at $\rho=a$. It follows from (17) that this plastic collapse mechanism occurs at $p=p_{k}$ where

$$
p_{k}=-\frac{2}{\sqrt{4-\eta^{2}}}
$$

It is obvious that $p_{k}$ is constant. Therefore, in the $p \tau$-space, this plastic collapse mechanism is interpreted as a straight line parallel to the $\tau$-axis. This line is illustrated in Figure 3 for $v=0.3$ and $G=H=F$. The curve corresponding to the initiation of plastic yielding (curve 1 ) is tangent to this line at point $k$.

In order to determine the curve corresponding to the other plastic collapse mechanism, it is necessary to solve Equation (16) for $\varphi_{m}$ numerically assuming that the value of $\varphi_{a}$ is given. Then, the value of $p$ immediately follows from Equation (17). Excluding $A$ and $B$ in Equation (15) yields

$$
\tau=(1+v)\left[\frac{\cos \varphi_{m}}{\sqrt{4-\eta^{2}}}\left(1-\frac{\eta \eta_{1}}{2}\right)-\frac{\eta_{1}}{2} \sin \varphi_{m}\right]-(1-v)\left[\frac{\cos \varphi_{m}}{\sqrt{4-\eta^{2}}}\left(1+\frac{\eta \eta_{1}}{2}\right)+\frac{\eta_{1}}{2} \sin \varphi_{m}\right]
$$

Since the value of $\phi_{m}$ has been found, the corresponding value of $\tau$ can be determined from this equation with no difficulty. Thus, the dependence of $p$ on $\tau$ is obtained in

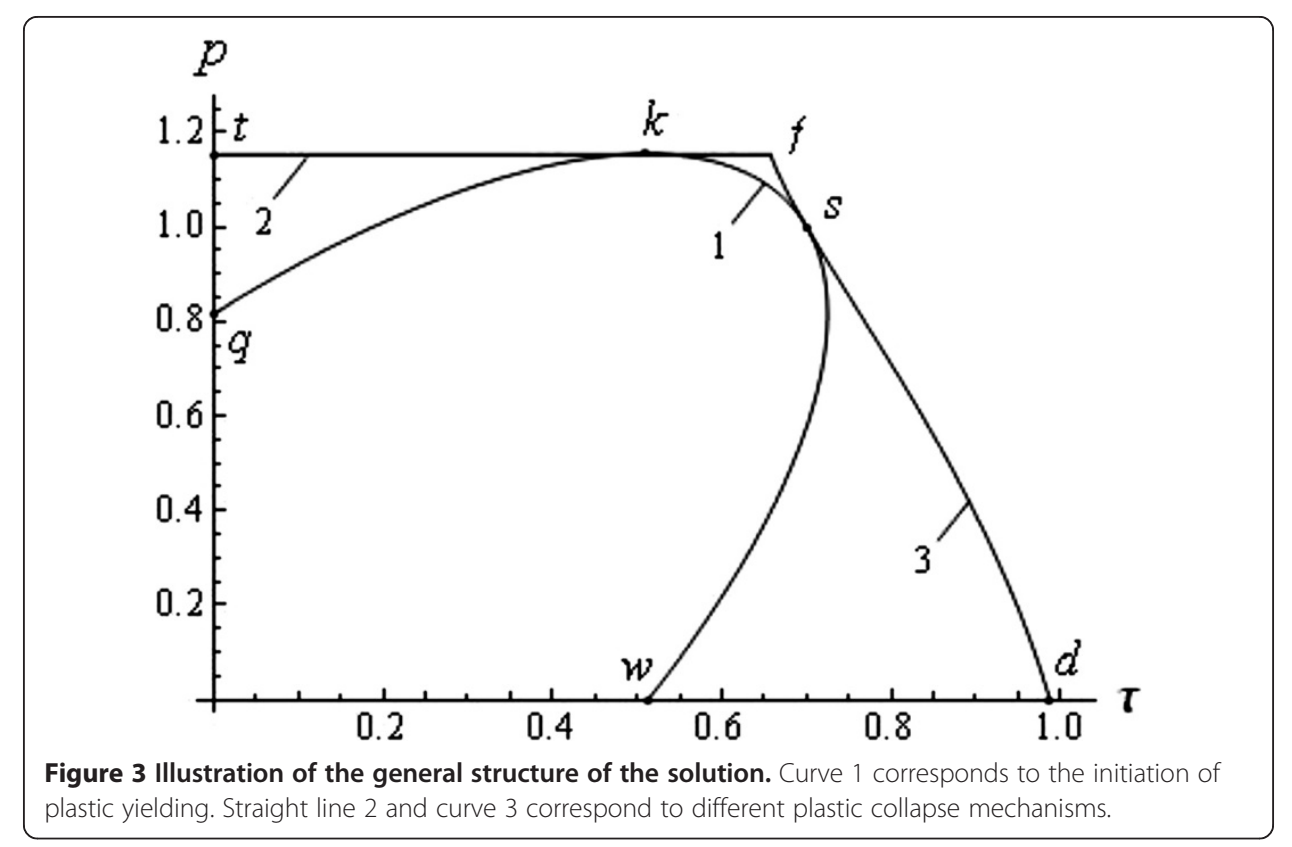


parametric form. This dependence is illustrated by curve 3 in Figure 3 for $a=1 / 2, v=0.3$ and $G=H=F$. Curves 1 and 3 have the same tangent line at point $s$.

\section{Results and discussion}

Figure 3 is a geometric illustration of the state of plastic collapse. In what follows, it is assumed that the increase in the loading parameters does not lead to unloading. It is convenient to divide curve 1 into three segments, namely $q k, k s$ and $s w$. If the initiation of plastic yielding occurs at some point of the segment $q k$, then the only possible mechanism of plastic collapse is localization of plastic deformation at $\rho=a$. In Figure 3, this mechanism of plastic collapse corresponds to a point of the line $t k$. If the initiation of plastic yielding occurs at some point of the segment $s w$, then the only possible mechanism of plastic collapse is the fully plastic disk. In Figure 3, this mechanism of plastic collapse corresponds to a point of the curve $s d$. Finally, if the initiation of plastic yielding occurs at some point of the segment $k s$, then either mechanism of plastic collapse is possible. Moreover, both mechanisms occur simultaneously if the values of the loading parameters correspond to point $f$. A special feature of the solution corresponding to point $k$ is that the plastic collapse occurs by strain localization when the entire disk is elastic (except the line $\rho=a$ ). A special feature of the solution corresponding to point $s$ is that the entire disk becomes plastic at the values of the loading parameters corresponding to the initiation of plastic yielding.

\section{Conclusions}

A new semi-analytical solution for the state of plastic collapse of a thin annular plastically orthotropic disk subject to thermo-mechanical loading has been found. The numerical part of the solution reduces to solving Equation (16) for $\varphi_{m}$. Plastic yielding is controlled by Hill's quadratic orthotropic criterion. The study has emphasized qualitative features of the plastic collapse solution whose general structure is illustrated in Figure 3. It has been shown that there are two plastic collapse mechanisms. According to one of these mechanisms, the load-bearing capacity of the disk is lost because of strain localization at its inner radius. According to the other plastic collapse mechanism, the entire disk becomes plastic. In addition to these two general cases, there are three special cases of great interest for both numerical solutions of similar problems and the interpretation of elastic/plastic stress solutions for thin plastically anisotropic structures. These special cases are denoted by symbols $k, s$ and $f$ in Figure 3. If the state of stress corresponds to point $k$, then the disk losses its load-bearing capacity by plastic localization at its inner radius whereas the entire disk (except the inner radius) is elastic. If the state of stress corresponds to point $s$, then the disk becomes plastic at the same values of the loading parameters at which plastic yielding initiates. A distinguished feature of point $f$ is that both of the aforementioned plastic collapse mechanisms occur simultaneously. It is expected that these qualitative features of the solution are rather common for a class of plastically anisotropic thin-walled structures and they can cause some difficulties with finding numerical solutions for such structures. 


\section{Authors' contributions}

SA formulated the boundary value problem and performed its qualitative analysis. CP performed numerical simulation. Both authors read and approved the final manuscript.

\section{Acknowledgements}

The research described in this paper has been supported by RFBR (Russia) and VAST (Vietnam), Project RFBR-14-01-93000.

\section{Author details}

'Laboratory for Strength and Fracture of Materials and Structures, A.Ishlinskii Institute for Problems in Mechanics of Russian Academy of Sciences, Moscow 119526, Russia. ${ }^{2}$ VAST, Institute of Mechanics, 264 Doi Can, Hanoi, Vietnam.

\section{Received: 22 November 2013 Accepted: 23 January 2014}

Published: 29 April 2014

\section{References}

1. Hsu YC, Forman RG (1975) Elastic-plastic analysis of an infinite sheet having a circular hole under pressure. Trans ASME J Appl Mech 42:347-352

2. Guven U (1992) Elastic-plastic annular disk with variable thickness subjected to external pressure. Acta Mechanica 92:29-34

3. Gamer U (1992) A concise treatment of the shrink fit with elastic-plastic hub. Int I Solids Struct 29:2463-2469

4. Lippmann H (1992) The effect of a temperature cycle on the stress distribution in a shrink fit. Int J Plast 8:567-582

5. Mack W (1993) Thermal assembly of an elastic-plastic hub and a solid shaft. Arch Appl Mech 63:42-50

6. Mack W, Bengeri M (1994) Thermal assembly of an elastic-plastic shrink fit with solid inclusion. Int J Mech Sci 36:699-705

7. Ball DL (1995) Elastic-plastic stress analysis of cold expanded fastener holes. Fatig Fract Eng Mater Struct 18:47-63

8. Poussard C, Pavier MY, Smith DJ (1995) Analytical and finite element predictions of residual stresses in cold worked fastener holes. J Strain Anal Eng Des 30:291-304

9. Alexandrov S, Alexandrova N (2001) Thermal effects on the development of plastic zones in thin axisymmetric plates. J Strain Anal Eng Des 36:169-176

10. Debski R, Zyczkowski M (2002) On decohesive carrying capacity of variable-thickness annular perfectly plastic disks. Zeitschrift für Angewandte Mathematik und Mechanik (ZAMM) 82:655-669

11. Alexandrova N, Alexandrov S (2004) Elastic-plastic stress distribution in a rotating annular disk. Mech Base Des Struct Mach 32:1-15

12. Alexandrova N, Alexandrov S (2004) Elastic-plastic stress distribution in a plastically anisotropic rotating disk. Trans ASME J Appl Mech 71:427-429

13. Gupta VK, Singh SB, Chandrawat HN, Ray S (2005) Modeling of creep behaviour of a rotating disc in the presence of both composition and thermal gradients. Trans ASME J Appl Mech 127:97-105

14. You LH, You XY, Zhang JJ, Li J (2007) On rotating circular disks with varying material properties. Zeitschrift für Angewandte Mathematik und Mechanik (ZAMM) 58:1068-1084

15. Jang JS, Kim DW (2008) Re-cold expansion process simulation to impart the residual stresses around fastener holes in 6061 A-T6 aluminium alloy. Proc IME B J Eng Manufact 222:1325-1332

16. Deepak D, Gupta VK, Dham AK (2009) Impact of stress exponent on steady state creep in a rotating composite disc. J Strain Anal Eng Des 44:127-135

17. Alexandrov SE, Lomakin EV, Jeng Y-R (2010) Effect of the pressure dependency of the yield condition on the stress distribution in a rotating disk. Doklady Physics 55:606-608

18. Chakherlou TN, Yaghoobi A (2010) Numerical simulation of residual stress relaxation around a cold-expanded fastener hole under longitudinal cyclic loading using different kinematic hardening models. Fatig Fract Eng Mater Struct 33:740-751

19. Masri R, Cohen T, Durban D (2010) Enlargement of a circular hole in a thin plastic sheet: Taylor-Bethe controversy in retrospect. Q Mech Appl Math 63:589-616

20. Alexandrov S, Jeng Y-R, Lomakin E (2011) Effect of pressure-dependency of the yield criterion on the development of plastic zones and the distribution of residual stresses in thin annular disks. Trans ASME J Appl Mech 78:031012

21. Kleiber M, Kowalczyk P (1996) Sensitivity analysis in plane stress elasto-plasticity and elasto-viscoplasticity. Comput Meth Appl Mech Eng 137:395-409

22. Hill R (1950) The mathematical theory of plasticity. Oxford University Press, Oxford

doi:10.1186/2196-1166-1-7

Cite this article as: Alexandrov and Pham: Plastic collapse mechanisms in thin disks subject to thermo-mechanical loading. Asia Pacific Journal on Computational Engineering 2014 1:7. 